\title{
Politique
}

\section{Dynamique et contraintes des finances publiques au Québec}

\section{André Blais et Kenneth McRoberts}

Numéro 3, hiver 1983

La crise des finances publiques au Québec

URI : https://id.erudit.org/iderudit/040417ar

DOI : https://doi.org/10.7202/040417ar

Aller au sommaire du numéro

Éditeur(s)

Société québécoise de science politique

ISSN

0711-608X (imprimé)

1918-6584 (numérique)

Découvrir la revue

Citer cet article

Blais, A. \& McRoberts, K. (1983). Dynamique et contraintes des finances publiques au Québec. Politique, (3), 27-62. https://doi.org/10.7202/040417ar d'utilisation que vous pouvez consulter en ligne.

https://apropos.erudit.org/fr/usagers/politique-dutilisation/ 


\title{
Dynamique et contraintes des finances publiques au Québec*
}

\author{
André BLAIS \\ Université de Montréal \\ Kenneth McROBERTS \\ Université York
}

Le thème de ce numéro prend pour acquis l'existence d'une «crise» des finances publiques. Il est de bon ton, par les temps qui courent, de parler de crise. Mais qu'est-ce qu'une crise exactement et de quelle crise s'agit-il au juste? On oublie en effet que cette crise nous était annoncée depuis longtemps. Dès le début des années 70, O'Connor écrivait son fameux livre, The Fiscal Crisis of the State ${ }^{1}$. Le problème c'est que le terme peut avoir plusieurs sens. Il renvoie parfois à l'idée de difficultés passagères, parfois à l'idée d'une grave impasse. Il signifie tantôt des perturbations, tantôt une rupture. Il nous apparaît plus approprié d'utiliser le terme dans un sens plus étroit de rupture par rapport à une pratique établie. Il y aura crise des finances publiques si le mode habituel de financement du gouvernement ne suffit plus ou est remis en question, soit

*Nous remercions Richard Nadeau, François Vaillancourt, Iain Gow, Philippe Faucher, Robert Young, Vincent Lemieux et Jean Crête pour leurs commentaires sur une première version de ce texte.

1. James O'CONNOR, The Fiscal Crisis of the State. (New-York, St. Martin's Press, 1973). 
qu'il doive assumer de nouvelles fonctions qui nécessitent des déboursés considérables, soit que les coûts de gestion des programmes augmentent rapidement, soit qu'il y ait révolte des contribuables. Avant même de discourir sur la portée de cette «crise», sur ses «véritables» causes, sur ses contradictions «profondes», sur ses répercussions à court, à moyen et à long terme, il est utile d'établir dans un premier temps un certain nombre de faits et de les situer dans leur contexte. Tel est l'objectif principal de ce texte. Il s'agit de présenter la situation des finances publiques québécoises, ce cerner son évolution récente, de la comparer à celle des autres provinces canadiennes. Ce tableau d'arrière-plan devrait nous permettre d'identifier les contraintes auxquelles le gouvernement peut difficilement échapper. Nous pourrons ensuite examiner les choix qu'il a opérés, de façon à dégager les stratégies qui semblent avoir été adoptées.

\section{La taille du gouvernement}

Un premier élément à considérer est l'ampleur des dépenses du gouvernement du Québec depuis 1960. Le tableau 1 présente les données pertinentes à ce sujet. L'indicateur retenu est la part des dépenses du gouvernement dans le produit intérieur brut. Nous avons choisi deux autres provinces (colonnes 3 et 4): l'Ontario, la grande voisine et concurrente à laquelle le Québec est comparé le plus fréquemment et la Saskatchewan, la province qui compte la plus longue tradition socialdémocrate, un gouvernement CCF-NPD ayant été au pouvoir de 1948 à 1964 et de 1971 à 1982. Les résultats pour l'ensemble des provinces canadiennes, le Québec exclu (colonne 2), sont également donnés.

Commençons par ces derniers. En termes relatifs, le niveau de dépenses a à peu près doublé. C'est entre 1965 et 
TABLEAU 1

Le niveau des dépenses ${ }^{1}$ du gouvernement québécois et des autres gouvernements provinciaux au Canada.

\begin{tabular}{|c|c|c|c|c|}
\hline Année financière & $\begin{array}{l}\text { Dépenses générales } \\
\text { brutes du gouver- } \\
\text { nement en } \% \text { du } \\
\text { produit intérieur } \\
\text { brut } \\
\text { QUÉBEC }\end{array}$ & $\begin{array}{l}\text { Dépenses générales } \\
\text { brutes du gouver- } \\
\text { nement en } \% \text { du } \\
\text { produit intérieur } \\
\text { brut } \\
\text { (autres prov. can.) }\end{array}$ & $\begin{array}{l}\text { Dépenses générales } \\
\text { brutes du gouver- } \\
\text { nement en \% du } \\
\text { produit intérieur } \\
\text { brut } \\
\text { ONTARIO }\end{array}$ & $\begin{array}{c}\text { Dépenses générales } \\
\text { brutes du gouver- } \\
\text { nement en \% du } \\
\text { produit intérieur } \\
\text { brut } \\
\text { SASKATCHEWAN }\end{array}$ \\
\hline $\begin{array}{l}1961-62 \\
1962-63 \\
1963-64 \\
1964-65 \\
1965-66 \\
1966-67 \\
1967-68 \\
1968-69 \\
1969-70\end{array}$ & $\begin{array}{r}9.7 \\
10.3 \\
11.2 \\
13.0 \\
13.6 \\
14.0 \\
15.0 \\
15.3 \\
16.2\end{array}$ & $\begin{array}{r}9.5 \\
10.0 \\
9.8 \\
9.8 \\
10.3 \\
11.4 \\
12.7 \\
13.5 \\
14.4\end{array}$ & $\begin{array}{r}7.6 \\
8.5 \\
8.2 \\
8.0 \\
8.5 \\
9.4 \\
10.7 \\
11.7 \\
12.5\end{array}$ & $\begin{array}{l}13.6 \\
12.1 \\
11.8 \\
13.1 \\
13.1 \\
13.1 \\
15.6 \\
16.1 \\
15.9\end{array}$ \\
\hline $\begin{array}{l}1970-71^{2} \\
1971-72 \\
1972-73 \\
1973-74 \\
1974-75 \\
1975-76 \\
1976-77 \\
1977-78 \\
1978-79 \\
1979-80\end{array}$ & $\begin{array}{l}19.0 \\
21.0 \\
21.0 \\
20.6 \\
22.0 \\
24.0 \\
24.2 \\
25.0 \\
25.8 \\
26.0\end{array}$ & $\begin{array}{l}16.4 \\
17.2 \\
16.7 \\
16.4 \\
17.5 \\
19.1 \\
18.6 \\
18.9 \\
18.7 \\
18.7\end{array}$ & $\begin{array}{l}14.6 \\
15.4 \\
14.9 \\
14.9 \\
16.0 \\
17.3 \\
16.7 \\
16.8 \\
16.9 \\
15.9\end{array}$ & $\begin{array}{l}18.4 \\
17.7 \\
19.1 \\
17.0 \\
17.2 \\
18.6 \\
19.9 \\
21.7 \\
20.4 \\
20.7\end{array}$ \\
\hline
\end{tabular}

1. Les dépenses découlant du régime de rentes du Québec et du Canada sont exclues.

2. Les données après 1970 ne sont pas strictement comparables à celles avant 1970 .

Sources: Statistique Canada, Finances publiques provinciales, cat. 68-207. Statistique Canada, Comptes économiques provinciaux, cat. 13-213. 
1972 que la progression a été la plus forte. Depuis 1975, il y a plafonnement et même une petite réduction depuis 1977. L'Ontario, pour sa part, a toujours été sous la moyenne canadienne mais les tendances y sont les mêmes. L'évolution des dépenses en Saskatchewan est passablement différente. Au tout début des années 60 , le budget est beaucoup plus élevé, reflétant l'orientation social-démocrate du gouvernement ${ }^{2}$. Pendant l'intervalle libéral, les dépenses n'augmentent que très peu; la province rejoint à peu près la moyenne canadienne en 1971. Depuis le retour du NPD, les dépenses ont remonté quelque peu, de sorte qu'il y a maintenant un écart de $2 \%$ avec l'ensemble des provinces "anglophones", mais là aussi la tendance depuis 1977 est à une légère diminution.

Le pattern québécois est particulièrement intéressant. Au départ le Québec ne se distingue guère du reste du Canada. La Révolution tranquille a cependant entraîné une augmentation sensible du budget provincial ${ }^{3}$, l'écart se situant à $3 \%$ en 1965. La fin des années 60 donne lieu à une halte: il y a croissance des dépenses mais la différence avec le reste du Canada s'atténue quelque peu. Puis dans la décennie 70 le mouvement reprend de plus bel. L'écart avec le reste du Canada passe de $3 \%$ à $7 \%$.

On pourrait multiplier les comparaisons. Peut-être faudrait-il souligner que le plafonnement des dépenses observé dans le reste du Canada (et qui s'applique également au gouvernement fédéral) ne constitue pas une anomalie: de 1977 à 1979, la part moyenne des recettes publiques dans le produit intérieur brut des pays membres de l'O.C.D.E. est à peu près

2. T. K. SHOYAMA, «Public Services and Regional Development in Canada ", Journal of Economic History, 26 (1978), 504.

3. Daniel LATOUCHE, «La vraie nature de... la révolution tranquille». Revue canadienne de science politique 7, (septembre 1974): 525-536. 
stationnaire ${ }^{4}$. Sur ce plan, l'évolution québécoise apparaît donc exceptionnelle.

Ces premiers chiffres semblent justifier à priori la position du gouvernement québécois, selon laquelle il faut freiner la croissance des dépenses: on dépense beaucoup plus qu'auparavant et qu'ailleurs, la part des dépenses gouvernementales dans le produit intérieur brut doit cesser d'augmenter. Il ne faudrait cependant pas conclure trop rapidement. Les actions passées ou le comportement des autres gouvernements ne sauraient en effet constituer un critère de référence absolu. Il faut plutôt considérer les facteurs qui incitent le gouvernement québécois, comme les autres, à vouloir freiner les dépenses. La raison première est évidente: il devient de plus en plus difficile pour les gouvernements d'augmenter leurs revenus. Le gouvernement québécois dispose de quatre sources de financement: les impôts, les recettes provenant de droits et permis ou de ventes ou de transferts des sociétés d'État, les transferts du gouvernement fédéral et finalement les emprunts.

Commençons par les transferts du gouvernement fédéral. Ils devraient atteindre les $5 \$$ milliards pour l'année financière 1982-1983. Ces transferts échappent au contrôle du gouvernement provincial, puisqu'ils relèvent d'ententes fédéralesprovinciales, dont le contenu dépend finalement du gouvernement fédéral. La croissance de ces transferts a été de l'ordre de $10 \%$ au cours des deux dernières années, un niveau inférieur à la croissance des dépenses, ce qui a forcé le gouvernement québécois à aller chercher de l'argent ailleurs.

Viennent ensuite les recettes non fiscales provenant de droits, permis, ventes ou transferts de sociétés d'État. Ces re-

4. En fait, la tendance d'ensemble demeure problématique. Le ratio est stationnaire de 1977 à 1979 mais on observe une remontée en 1980. Les estimations provisoires de 1981 vont aussi dans le sens d'une hausse. Voir O,C,D.E., Statistiques des recettes publiques des pays membres de l'O.C.D.E., 1965-1981. 
cettes devraient dépasser 1,6 \$ milliard en 1982-1983 mais demeurent marginales dans l'ensemble des revenus du gouvernement. On voit mal comment ces recettes pourraient être augmentées considérablement. Une initiative comme la redevance exigée de l'Hydro-Québec n'a rapporté que très peu. Toute hausse des dépenses ne peut donc être financée que par une augmentation des impôts ou des emprunts.

Une hausse d'impôts apparaît pour le moins difficile. Le fardeau fiscal des Québécois est beaucoup plus lourd que celui des résidents de l'Ontario: l'écart a été estimé à $14 \%$ par le Ministre des finances du Québec ${ }^{5}$. Sur ce plan, le Québec «devance» nettement toutes les autres provinces ${ }^{6}$.

Selon la théorie du «vote avec les pieds», le contribuable peut résister à une hausse d'impôt en quittant ou en menaçant de quitter le pays, la province ou la municipalité qui impose un fardeau fiscal trop élevé. Cette réaction ou cette menace forcerait les gouvernements à se limiter à des niveaux de taxation similaires à ceux prévalant dans les pays, provinces ou municipalités voisines. C'est ce type de préoccupation qui avait amené la Commission Bélanger, dans les années 60, à recommander pour le Québec un régime fiscal qui ne soit pas trop différent de celui du reste du Canada et des États-Unis. Cette théorie a certes une dose de validité. Au Québec, cependant, la mobilité (surtout chez les francophones) est faible. La marge de manœuvre du gouvernement est ainsi plus grande.

Il n'en demeure pas moins que la résistance des contribuables face à une augmentation de leur fardeau fiscal est très réelle. Les signes de cette résistance sont clairs. Aux États-

5. Gouvernement du Québec, Budget 1982-1983. Renseignements supplémentaires, annexe III.

6. Allan J. MacEACHEN, «Federal-Provincial Fiscal Arrangements in the Eighties ", Submission to the Parliamentary Task Force on the Federal-Provincial Fiscal Arrangements, 23 avril 1981. 
Unis, la proposition 13 en est l'exemple le plus percutant, mais d'autres propositions, comme la $2 \frac{1}{2}$ au Massachussetts, vont dans le même sens. Le mécontentement vis-à-vis le fardeau fiscal est de plus en plus généralisé: en 1978, seulement $20 \%$ des citoyens américains trouvaient les taxes «raisonnables », contre $30 \%$ au début des années $70^{7}$. Plusieurs études indiquent que cette volonté de réduire les taxes a été un facteur important dans le vote sur les différents référendums tenus en Californie, au Michigan et au Massachussetts ${ }^{8}$. En même temps, ces mêmes études révèlent une volonté tout aussi ferme de ne pas réduire les services offerts. Il se dégage un appui nettement majoritaire à la plupart des programmes gouvernementaux, surtout les programmes de portée générale ${ }^{9}$. Par ailleurs, de plus en plus de citoyens ont le conviction que le gouvernement est inefficace et gaspille de l'argent. En somme les gens en veulent autant pour moins cher. Ce souhait est peut-être utopique. Quoi qu'il en soit, la résistance à des hausses d'impôt ne fait guère de doute. Au Québec nous ne disposons pas de données comparables. L'État a probablement une plus grande légitimité. Les Québécois semblent un peu moins unanimes à croire au gaspillage des gouvernements ${ }^{10}$. Le fait que le fardeau fiscal soit beaucoup plus lourd qu'ailleurs ne laisse cependant pas, pouvons-nous penser, de marge de manœuvre supplémentaire au gouvernement.

7. Everett Carl LADD jr. et al., "The Polls: Taxing and Spending", Public Opinion Quarterly 13. (printemps 1979): 126-136.

8. Jack CITRIN, «Do People Want Something for Nothing: Public Opinion on Taxes and Government Spending". National Tax Journal 32, (juin 1979): 113131; Paul N. Courant et al., "Why Voters Support Tax Limitation Amendments: The Michigan Case». National Tax Journal 33, (mars 1980): 1-21; Helen F. LADD et Julie Boatright WILSON, "Why Voters Support Tax Limitations: Evidence from Massachussetts Proposition 21/2». National Tax Journal 35, (juin 1982): 121-149.

9. P. PERETZ, «There Was No Tax Revolt», Politics and Society, 11 (1982): 231-249.

10. Allan KORNBERG et al., Representative Democracy in the Canadian Provinces. (Scarborough, Prentice-Hall, 1982). 
La résistance des contribuables nous semble avoir deux sources principales. La première est la récession économique. La croissance de l'État a été grandement favorisée par la croissance économique. Cette croissance faisait en sorte que, même si les recettes fiscales augmentaient rapidement, le revenu privé (le "take home pay») continuait d'augmenter ${ }^{11}$. Maintenant, toute augmentation des impôts entraîne une diminution réelle du revenu privé. On comprend alors aisément que l'opposition soit plus forte. On observe également un certain désillusionnement vis-à-vis les possibilités de l'État. Certes le désillusionnement n'est pas total puisque rares sont ceux qui souhaitent des coupures radicales dans les services gouvernementaux. Mais on se montre maintenant plus sceptique et on reconnaît que si le marché a ses vices, l'intervention de l'État a également les siens.

La dernière source de financement possible est alors les emprunts. Depuis le début des années 80 , le déficit du gouvernement du Québec est de l'ordre de 3 \$ milliards. L'Ontario, dont le budget est du même ordre, prévoit un déficit de 2,2 \$ milliards en 1982-1983. Par contre, la dette du gouvernement québécois est quelque peu inférieure à celle du gouvernement ontarien (14\$ milliards contre $17 \$$ milliards au 31 mars 1982), en bonne partie parce que les déficits du Québec ont été plus faibles que ceux de l'Ontario au cours des années 70. Finalement, le service de la dette devrait représenter $8,6 \%$ des dépenses du gouvernement du Québec en 1982-1983, contre $9,5 \%$ en Ontario. Dans les deux cas, le pourcentage est à la hausse, mais de façon encore plus marquée au Québec. En somme, le Québec a pu profiter des faibles déficits réalisés précédemment mais cet avantage est maintenant disparu, de sorte

11. Richard ROSE et Guy PETERS, Can Government go Bankrupt? (NewYork, Basic Books, 1978). 
que c'est l'Ontario qui a pu récemment augmenter ses déficits à un niveau s'approchant de celui du Québec. Dans les deux provinces, le niveau d'endettement a augmenté considérablement.

Encore une fois, les chiffres ne sauraient dicter a eux seuls les choix politiques. Ils nous en disent long, cependant, sur les contraintes dont le gouvernement québécois, comme les autres gouvernements, doit tenir compte. On argumentera que les déficits du gouvernement fédéral sont encore plus importants. Cela est vrai mais ne devrait pas être considéré comme une justification. On ajoutera qu'en période de récession les déficits des gouvernements peuvent être augmentés, précisément pour résorber la crise. À cela, il faut répliquer qu'ils ont déjà connu une hausse considérable et qu'il convient de se demander s'il faut aller plus loin. De plus, on ne peut guère se fier aux gouvernement pour appliquer de façon rigoureuse la «solution keynésienne». Dans la perspective keynésienne, en effet, les gouvernements devraient réaliser des surplus en période de croissance économique, ce qu'ils ne font que rarement ${ }^{12}$. Il y a donc des limites aux déficits gouvernementaux. Ils ont le désavantage, en plus de reporter le fardeau fiscal aux générations futures, de restreindre la marge de manœuvre du gouvernement face aux milieux financiers qui ne manquent pas de poser certaines conditions, lors de l'octroi ou du renouvellement d'emprunts ${ }^{13}$.

12. Voir l'article de James Iain GOW, «Perspectives historiques sur les compressions budgétaires» et J. Harvey PERRY, Background of Current Fiscal Problems. (Toronto, Canadian Tax Foundation, 1982), 9.

13. Selon Jacques PARIZEAU, les milieux financiers auraient exercé des pressions en 1966 pour que le Québec se montre plus généreux à l'endroit de l'Université McGill (Le Devoir, 12 février 1970, cité par Ted GERRARD, "Quebec/Ontario Access to Capital Markets, 1867-1980», miméo, York University). Plus récemment l'ampleur des déficits (de même que la réduction des transferts fédéraux) a été invoquée pour justifier la baisse de la cote de crédit du gouvernement (Le Devoir, 17 juillet $1982,5)$. 
En somme, la dette du gouvernement québécois, sans être catastrophique, est suffisamment élevée pour inciter à une grande prudence. En 1980 et 1981, les emprunts nets totaux du secteur public ont excédé les investissements. Rien ne sert non plus d'exagérer: le seuil de $3 \$$ milliards n'a en soi rien de magique et n'est pas infranchissable ${ }^{14}$. Il reste que pour l'essentiel, le niveau d'endettement du gouvernement québécois ne saurait être substantiellement majoré sans hypothéquer sérieusement la marge de manœuvre du gouvernement dans les années à venir.

La conclusion est donc la même pour chacune des quatre sources de financement: les possibilités d'accroittre les fonds apparaissent bien minces. De ce point de vue, le gouvernement québécois semble avoir peu de choix. Il lui faut plafonner les dépenses. Cette situation n'a en soi rien de dramatique. Il s'agit de s'assurer que les dépenses n'augmentent pas plus rapidement que le produit intérieur brut ou le revenu personnel. A l'intérieur de ce paramètre général, par ailleurs, le gouvernement conserve une certaine marge de manœuvre. Il peut augmenter ou diminuer certains impôts et réaménager la ventilation de ses dépenses. Nous allons examiner brièvement chacun de ces deux aspects en situant d'abord la position du Québec par rapport au reste du Canada, en identifiant ensuite les principales modifications qui ont été apportées dans les récents budgets et en tentant finalement de dégager la (ou les) logique(s) qui les sous-tend(ent).

\section{La stratégie fiscale}

Considérons d'abord les sources de revenu du gouvernement québécois et des autres gouvernements provinciaux en vembre 1982.

14. Alain DUBUC, "Déficit: quel est le point d'alarme? ", La Presse, 13 no- 
1979-1980 (dernière année pour laquelle des données complètes et comparables sont disponibles). Nous nous limiterons ici aux recettes fiscales, c'est-à-dire celles provenant d'impôts. Le tableau 2 indique que les deux principales sources de revenu sont l'impôt sur le revenu des particuliers et la taxe générale de vente. L'Ontario se démarque surtout par l'importance qu'y revêt la cotisation pour l'assurance-maladie, cotisation qui n'est en vigueur que dans trois provinces (Ontario, Alberta et la Colombie-Britannique). La Saskatchewan semble se distinguer au niveau de la taxe générale de vente mais les résultats de 1979 sont exceptionnels et ne sont guère significatifs: la taxe de vente n'y est en effet que de $5 \%$.

La fiscalité québécoise apparaît fort différente. Le fait le plus marquant est la part considérable qu'occupe l'impôt sur le revenu des particuliers. Cet impôt est responsable de plus de la moitié des recettes fiscales du gouvernement québécois: la proportion est à la hausse depuis le début des années 60. L'écart avec le reste du Canada provient surtout du fait que le Québec est la seule province où les taux et les exemptions ne sont pas indexés (cette indexation est en vigueur dans le reste du Canada depuis - 1974). Les taux d'imposition du Québec excèdent ceux de l'ontario d'environ $20 \%$ en 1979 , alors que l'écart n'était que d'à peu près $7 \%$ en $1975^{15}$. L'inflation (accompagnée de la non-indexation) fait en sorte que l'impôt à payer par le contribuable augmente considérablement même si le revenu réel est stable. Le gouvernement québécois a ainsi été l'un des grands bénéficiaires de l'inflation.

Pour les autres sources de revenus, la fiscalité québécoise apparaît moins originale. Les taux d'imposition (qui constituent un indicateur plus valable, mais qu'il serait trop long

15. Nicole BRUNET, "Etude comparative de quelques aspects du portrait fiscal des provinces au Canada», miméo, Université de Montréal, automne 1982. 
TABLEAU 2

Les recettes fiscales des gouvernements provinciaux au Canada (1979-80)

\begin{tabular}{l|c|c|c|c}
\hline \multicolumn{1}{c|}{ Source } & $\begin{array}{c}\text { Québec } \\
\%\end{array}$ & $\begin{array}{c}\text { Reste } \\
\text { du Canada } \\
\%\end{array}$ & $\begin{array}{c}\text { Ontario } \\
\%\end{array}$ & $\begin{array}{c}\text { Sas- } \\
\text { katchewan } \\
\%\end{array}$ \\
\hline rev. des particul. & 51.5 & 37.5 & 34.7 & 36.3 \\
bénéfices corp. & 6.7 & 13.0 & 11.9 & 10.7 \\
taxe gén. vente & 17.8 & 22.3 & 23.1 & 29.0 \\
taxe sur carburant & 5.2 & 7.0 & 7.0 & 10.1 \\
primes & 11.2 & 12.9 & 15.4 & 5.9 \\
autres & 7.7 & 7.1 & 7.9 & 7.9 \\
\hline & $100 \%$ & $100 \%$ & $100 \%$ & $100 \%$ \\
\hline
\end{tabular}

1. Comprend les primes et taxes d'assurance-maladie et les cotisations aux caisses des accidents de travail, mais exclut les cotisations aux régimes universels de rentes.

Source: Statistique Canada. Les finances publiques provinciales, cat. 68-207.

d'examiner ici en profondeur) sont les mêmes que ceux prévalant dans le reste du Canada ou, s'ils sont supérieurs, la différence n'est pas considérable. Par contre, l'impôt sur les bénéfices des sociétés et la taxe sur le carburant sont peu élevés, les taux (en 1979) étant légèrement inférieurs à ceux de l'Ontario. Signalons enfin que le Québec était la seule province, jusqu'à tout récemment, à prélever une cotisation des employeurs (pour l'assurance-maladie).

Il devient alors intéressant d'examiner les principaux changements annoncés par le gouvernement québécois au cours des deux dernières années. Dans le budget 1981-1982, le ministre des Finances se préoccupe surtout de la fiscalité des entreprises. Il introduit l'obligation pour les sociétés d'État de payer un dividende de $20 \%$ sur leurs profits ordinaires (avec des modalités particulières pour l'Hydro-Québec). Il modifie substantiellement la taxation des entreprises en augmentant la contribution des employeurs aux services de santé (de 1,5\% à 
$3,0 \%$ du salaire versé) de même que la taxe sur le capital (de $0,30 \%$ à $0,45 \%$ ) mais en réduisant par ailleurs le taux d'imposition sur les bénéfices des sociétés de $13 \%$ à $8 \%$ pour les grandes entreprises (et à $5,5 \%$ à partir de janvier 1983) et de $12 \%$ à $3 \%$ pour les petites entreprises. Ces modifications ont entraîné une hausse substantielle des impôts payés par les entreprises en 1981-1982 mais impliquent une légère baisse à moyen terme, c'est-à-dire à partir de janvier 1983. Notons enfin une hausse de la taxe sur le tabac, dont le taux est majoré de $40 \%$ à $45 \%$ (il sera fixé à $50 \%$ en 1982). Dans le budget supplémentaire de novembre 1981, c'est, on le sait, la taxe sur les carburants qui est touchée, le taux étant porté de $20 \%$ à $40 \%$. Finalement, le budget 1982-1983 annonce la hausse de la taxe de vente, de $8 \%$ à $9 \%$, pour une période de dix mois.

Nous avons laissé de côté jusqu'ici l'impôt sur le revenu des particuliers. La raison en est simple. Les modifications apportées ont été minimes et ne touchent que les frais de garde et le régime enregistré d'épargne-logement. Il convient cependant de considérer la question de l'indexation. Lorsqu'il n'y a pas indexation, l'inflation pénalise le contribuable de deux façons. Premièrement, même si le revenu réel est stationnaire, le revenu nominal augmente, ce qui place le contribuable dans une catégorie plus élevée, le forçant ainsi à payer davantage d'impôt. Deuxièmement, puisque les déductions et exemptions ne sont pas indexées, le revenu imposable augmente plus rapidement que le revenu total ${ }^{16}$. C'est ce qui a amené le gouvernement canadien, depuis 1974, à indexer de façon presque complète le régime d'imposition. Ce système est en vigueur dans tout le reste du Canada. Au Québec, le gouvernement adopte des mesures ponctuelles à chaque année. Lorsqu'il était

16. Robin W. BOADWAY et Harry M. KITCHEN, Canadian Tax Policy, (Toronto, Canadian Tax Foundation, 1980). 
dans l'opposition, le Parti Québécois réclamait l'indexation de l'impôt. Dans son premier budget, le ministre des Finances conclut que l'état des finances publiques ne permet pas de mettre tout de suite en application cette politique. Dans son second budget, il annonce qu'une indexation partielle des exemptions (mais non de la table) entre en vigueur à partir de l'année suivante (1979), mais elle a été retardée d'une autre année, suite à la dispute fédérale-provinciale sur la taxe de vente. En 1980, l'indexation a été de 6\%. En 1981 et 1982 elle a été de $7,5 \%$, avec en plus une «réduction» de $3 \%$ des taux en 1981. Une "réduction» supplémentaire de $2 \%$ avait été annoncée pour 1982, mais a été annulée dans le budget supplémentaire de novembre 1981. Le budget 1982-1983 prévoit une autre indexation, de 7,5\%, des exemptions, en janvier 1983, mais rien en ce qui concerne les taux. Ce qu'il faut retenir, c'est que l'impôt n'est que très peu indexé, ce qui constitue une hausse «déguisée». Ceci a permis aux recettes provenant de cet impôt d'augmenter de près de $15 \%$ par année. En Ontario, le gouvernement provincial a estimé que l'indexation mise en vigueur en 1974 lui coûtait 1,6 - milliards en $1980^{17}$. On comprend donc aisément la portée de la nonindexation pour le gouvernement québécois.

Plusieurs augmentations d'impôt nous ont donc été annoncées au cours des dernières années. Le gouvernement se soucie évidemment de les présenter en «temps opportun». Les «mauvaises» nouvelles arrivent juste après une élection, dans l'espoir qu'elles auront été oubliées dans quatre ans. Les budgets pré-référendaire et pré-électoral ne contenaient aucune hausse d'impôt, sauf pour les entreprises. Dès novembre 1981, toutefois, le gouvernement double la taxe sur les carburants et annule la réduction promise pour 1982 de la table de l'impôt

17. Le budget de l'Ontario, 1981, document budgétaire « $\mathrm{A} », 8$. 
personnel. De plus, les «bonnes» nouvelles sont annoncées longtemps à l'avance alors que les «mauvaises» entrent en vigueur immédiatement. La réforme de la fiscalité des entreprises entraînait, par exemple, une hausse considérable d'impôt la première année, qui était rendue acceptable par une légère diminution par la suite. De même, l'augmentation de la taxe de vente «se digère» d'autant plus facilement qu'elle est temporaire.

Par ailleurs, les choix qui ont été faits nous semblent refléter un certain nombre de préoccupations. Une première est la plus grande attention accordée aux taxes à la consommation. Plusieurs facteurs nous semblent devoir rendre compte de cette évolution. Les taxes sur les biens «nocifs» comme le tabac et l'alcool peuvent facilement être présentées comme allant dans «l'intérêt public», de telle sorte qu'il est bien difficile pour les groupes touchés de s'y opposer. Dès le départ, les défenseurs du tabac et de l'alcool (y compris la bière) sont suspects. Il n'y a donc guère de risque pour un gouvernement de hausser ces taxes. Par contre, l'augmentation de la taxe sur les carburants, qui polluent notre environnement, a suscité beaucoup de remous. Cette réaction peut s'expliquer par le niveau beaucoup plus brutal de la hausse et par le fait que l'automobile est probablement considérée comme un bien «essentiel», en particulier pour le travail. Le ministre des Finances a peut-être jugé que les Québécois avaient quatre ans pour s'habituer à cette taxe qui était déjà relativement faible. Cette augmentation avait été d'ailleurs suggérée explicitement dans le rapport du «comité des onze» (sous la présidence de Gilbert Paquette), rapport qui critiquait certains points du budget Parizeau de mars 1981. Le ministre des Finances s'assurait ainsi de l'appui de ses ex-critiques.

Dans son dernier budget, le ministre des Finances a aussi annoncé une hausse de la taxe de vente. Il n'a pas été le seul à 
emprunter cette direction. Deux semaines auparavant, son collègue de l'Ontario avait décrété un élargissement considérable de la couverture (la taxe s'applique, entre autres, aux frais de main-d'œuvre pour l'installation, la réparation et l'entretien des automobiles, téléviseurs, appareils électroménagers). En 1981, le taux de la taxe a été augmenté en Colombie-Britannique et à l'île-du-Prince-Édouard. C'est donc là une source de revenu à laquelle les gouvernements provinciaux semblent vouloir puiser davantage. Cette évolution se produit au moment même où un débat important prend place dans le milieu de la fiscalité sur la pertinence relative de l'impôt sur le revenu et de l'impôt sur la consommation $^{18}$. Alors qu'auparavant le revenu était considéré comme le meilleur indicateur de la capacité de payer d'un contribuable, plusieurs soutiennent maintenant que le niveau de consommation est un élément plus valable et proposent une réforme complète de la fiscalité dans ce sens. Les gouvernements ne sont certes pas insensibles à ce débat et, à un moment où ils se préoccupent davantage de l'investissement, sont plus tentés de hausser les taxes à la consommation que l'impôt sur le revenu. Il est vrai que la taxe de vente a mauvaise presse parce qu'elle est jugée régressive. Cette perception n'est cependant pas valide, puisqu'au Québec le logement et l'alimentation sont exclus ${ }^{19}$. Si on tenait compte du revenu à long terme plutôt que du revenu annuel ${ }^{20}$, on peut supposer qu'elle serait même légèrement progressive. Il s'agit aussi d'une taxe dont

18. Joseph A. PECKMAN (dir.), What Should be Taxed: Income or Expenditure? (Washington, The Brookings Institute, 1980) et R. M. BIRD, "What Should We Tax: Income or Consumption?». Report of Proceedings of 30th Tax Conference, (Toronto, Canadian Tax Foundation, 1979): 219-225.

19. François VAILLANCOURT et Jacques BERTHIAUME, "A Comparative Analysis of the Incidence of Retail Sales Tax in Ontario and Québec, 1970». Canadian Tax Conference, (Toronto, Canadian Tax Journal 26. (septembre-octobre 1978): 596605.

20. P. MUSGRAVE et R. MUSGRAVE, Public Finance in Theory and Practice. (New-York, McGraw-Hill, 1976). 
les coûts d'administration sont peu élevés et pour laquelle la fraude est probablement peu répandue. Les principaux inconvénients se posent dans les régions frontières (Hull en particulier), les citoyens pouvant aller se procurer des biens moins lourdement taxés dans une autre province (ce qui provoque évidemment les protestations véhémentes des commerçants).

La hausse de la taxe de vente a été présentée comme étant temporaire. Il sera intéressant d'observer ce que le prochain budget nous réservera sur ce plan. Si les fonds disponibles se font rares, il ne serait pas étonnant que la hausse soit maintenue (indéfiniment, ou pour une autre année), d'autant plus que les contribuables sont maintenant habitués à la taxe de $9 \%^{21}$ ou que, comme en Ontario, la couverture soit élargie, d'autant plus que les commerçants, qui sont les plus directement touchés par cette taxe, ne semblent pas constituer un groupe très influent à un moment où le gouvernement québécois dit s'intéresser au "virage technologique».

Qu'en est-il par ailleurs des modifications apportées à la fiscalité des entreprises? Il y a d'abord, rappelons-le, la décision de réduire l'impôt sur les bénéfices et d'augmenter en contrepartie la taxe sur le capital et la contribution des employeurs aux services de santé. L'explication principale donnée par le ministre des Finances concerne les difficultés d'estimation du revenu lorsqu'une partie de la production origine de l'extérieur du Québec: «Dans un pays comme le Canada, où un grand nombre d'entreprises fonctionnent dans plusieurs provinces sinon dans toutes, la taxation des profits par chaque gouvernement provincial présente des problèmes considérables ${ }^{22}$. Ces considérations techniques sont certes pertinentes.

21. La thèse selon laquelle les gens finissent par accepter un certain niveau de taxation est exposée dans Alan J. PEACOCK et Jack WISEMAN, The Growth of Public Expenditure in the United Kingdom. (London, Allen et Unwin, 1967).

22. Gouvernement du Québec, Budget 1981-1982, Discours sur le budget, 25. 
Il est cependant douteux qu'elles aient eu un poids déterminant. Dans les autres provinces canadiennes par exemple, le taux d'imposition des grandes entreprises n'a pas été diminué au cours des dernières années: il a même augmenté dans quatre provinces depuis 1980 (Terre-Neuve, Nouvelle-Écosse, Nouveau-Brunswick et Colombie-Britannique). On peut supposer que le calcul a plutôt été de faire porter une partie du poids par le gouvernement fédéral. La taxe sur le capital et la contribution des employeurs, en effet, sont déductibles du revenu imposable, de sorte que près de la moitié du coût devra être absorbée par le gouvernement fédéral. Le truc est astucieux, il fallait y penser! À noter que jusqu'à tout récemment le Québec était la seule province où les employeurs payaient des cotisations. Les autres provinces pourraient être tentées de suivre l'exemple. Déjà le gouvernement manitobain a agi dans ce sens dans son budget 1982 .

L'impôt sur les bénéfices des sociétés est d'ailleurs l'impôt le plus débattu présentement, surtout en raison de la prise en compte de l'inflation dans le calcul du profit ${ }^{23}$. Les pressions pour l'abolition (ou tout au moins la réduction) de cet impôt sont fortes ${ }^{24}$. Par ailleurs, le gouvernement introduit un taux réduit pour la petite entreprise. Ce faisant, le Québec suit l'exemple donné par huit autres provinces et le gouvernement fédéral. L'organisation et la mobilisation des intérêts de la petite entreprise ${ }^{25}$ ne sont certes pas étrangères à cette évolution. Mais il faut aussi souligner que les gouvernements provinciaux

23. Plusieurs articles traitant de cette question dans Wayne R. THIRSK et John WHALLEY (dir.), Tax Policy Options in the 1980s. (Toronto, Canadian Tax Foundation, 1982).

24. Voir Richard M. BIRD, Taxing Corporations. (Montréal, The Institute for Research on Public Policy, 1980). Plus récemment l'économiste Pierre FORTIN faisait des recommandations dans le même sens (Le Devoir, 31 mars 1982).

25. James GILLIES, Where Business Fails. (Montréal, The Institute of Research on Public Policy, 1981), XII, note 2. 
(et le gouvernement fédéral d'ailleurs) n'ont pas intérêt à trop diminuer le taux d'imposition des grandes entreprises, pour autant qu'une bonne partie d'entre elles sont étrangères (surtout américaines). Deutsch et Jenkins ${ }^{26}$ ont en effet montré que les montants épargnés par les entreprises risquent de se retrouver au Trésor américain, puisque les impôts payés par ces entreprises au gouvernement provincial peuvent être déduits de l'impôt à payer au gouvernement américain. Cette considération, qui ne semble pas avoir été prise en compte par le ministre des Finances, jette un doute sérieux sur la pertinence de réduire aussi fortement le taux d'imposition des grandes entreprises. Le ministre des Finances de l'Ontario semble l'avoir compris, lui qui annonçait dans son dernier budget la suspension pour deux ans de l'impôt sur les bénéfices des petites entreprises et le maintien du taux en vigueur pour les grandes entreprises.

Il ne faudrait pas en conclure que ces modifications avantagent automatiquement les entreprises. Pour 1981, en effet, la hausse de la contribution des employeurs et celle de la taxe sur le capital constituent une augmentation du fardeau fiscal des entreprises privées d'environ $350 \$$ millions, alors que la réduction de l'impôt sur le revenu ne représente que $140 \$$ millions. Par contre ce dernier montant devrait dépasser le 400 \$ millions à partir de 1983 , de sorte qu'il semble y avoir un léger allègement du fardeau fiscal à partir de ce moment ${ }^{27}$. Alors que le fardeau fiscal des entreprises québécoises et ontariennes était à peu près similaire jusqu'en 1980, l'écart se situe à environ $10 \%$ à partir de 1983 (la moitié de cet écart découle de la décision du gouvernement ontarien d'exempter d'impôt

26. Antal DEUTSH et Glenn P. JENKINS, «Tax Incentives, Revenue Transfers and the Taxation of Income from Foreign Investment », dans THIRSK et WHALLEY (dir.), op. cit. nexe III.

27. Gouvernement du Québec, Budget 1981-1982: Discours sur le budget, an- 
les petites entreprises en 1982 et 1983) ${ }^{28}$. Dans l'ensemble, donc, les entreprises ne sont pas plus ou moins bien traitées qu'auparavant. Il s'agit plutôt d'une restructuration qui a pour effet de donner plus d'argent au gouvernement provincial et moins au gouvernement fédéral.

Considérons finalement l'impôt sur le revenu des particuliers. Sur ce plan, le gouvernement du Québec a beau jeu. Le fait que les tables, ainsi que les déductions et exemptions, ne sont à peu près pas indexées assure une croissance des recettes de l'ordre de $15 \%$ par an. Politiquement, les avantages sont évidents. Pour obtenir les mêmes résultats, l'Ontario doit augmenter son taux alors que le ministre des Finances nous parle d'une «baisse» d'impôt, qui est toute artificielle. Cette situation a donné au gouvernement du Québec une marge de manœuvre appréciable, qu'il a exploitée au maximum. Tout indique en effet que l'impôt sur le revenu des particuliers a été utilisé jusqu'ici comme un coussin utile pouvant absorber une partie du manque à gagner du gouvernement. La structure de présentation du budget 1981-1982 nous semble de ce point de vue révélatrice. Le ministre des Finances y aborde d'abord les principaux changements apportés à la fiscalité (surtout celle des entreprises) mais ne considère l'impôt sur le revenu des particuliers qu'après avoir examiné "la compression des dépenses, et seulement ensuite on indiquera dans quelle mesure et jusqu'où l'on pense pouvoir pousser la réduction du fardeau fiscal des contribuables ${ }^{29}$. En somme le niveau d'indexation est établi, une fois que le reste du budget est arrêté, pour combler la différence.

Pour être complet il faudrait, il est vrai, tenir compte des abris fiscaux. Depuis 1979, le Québec offre un régime

28. Gouvernement du Québec, Budget 1982-1983: Renseignements supplémentaires, annexe III.

29. Gouvernement du Québec, Budget 1981-1982. Discours sur le budget, 27. 
d'épargne-actions qui a pour conséquence de réduire sensiblement l'écart entre le Québec et l'Ontario, en particulier pour ceux qui ont des revenus élevés. L'utilisation de cet abri fiscal est ingénieuse. À ceux qui se plaignent d'un fardeau fiscal trop lourd, le gouvernement peut répondre que cette mesure, qui a aussi pour effet d'accroître l'investissement au Québec, permet de payer un impôt «raisonnable», d'autant plus que le coût de la vie est moins élevé à Montréal qu’à Toronto ${ }^{30}$. En même temps cet abri fiscal ne semble pas avoir été «trop» exploité, de sorte que les revenus de l'État ne sont pas beaucoup affectés ${ }^{31}$. Les grands perdants sont probablement les classes moyennes qui connaissent peu cette mesure.

À plus d'un point de vue, on pourrait qualifier la stratégie fiscale du gouvernement québécois d'habile. Il a réussi à augmenter ses recettes sans soulever trop d'opposition. Il a profité de l'inflation, qui a gonflé les fonds provenant de l'impôt sur le revenu des particuliers sans qu'il n'ait à intervenir directement. Il est même parvenu à nous présenter l'indexation partielle des exemptions comme une "baisse" d'impôt, alors qu'il s'agit effectivement d'une hausse. Les augmentations de taxes les plus visibles ont touché des biens dont on s'entend généralement pour dire que "l'intérêt public» exige qu'ils soient moins consommés. L'augmentation de la taxe de vente a été présentée comme temporaire. La fiscalité des entreprises a été modifiée de façon à ce que celles-ci, tout en payant un impôt total à peu près similaire, en donnent plus au Québec et moins au gouvernement fédéral. Bien sûr, il y a quelques im-

30. J. M. COUSINEAU, R. LACROIX et F. VAILLANCOURT, Les marchés du travail de Montréal et de Toronto. (Montréal, Centre d'études en administration internationale, 1982).

31. Sur l'utilisation des abris fiscaux comme une soupape visant à faire accepter des impôts par ailleurs très lourds, voir Robert D. BROWN, «A Cristal Review of Tax Shelters: Loophole or Escape Hatch?» dans THIRSK et WHALLEY (dir.)., op. cit. 
pairs. La pertinence d'une réduction aussi marquée du taux d'imposition des grandes entreprises, entre autres, apparaît pour le moins douteuse. Mais dans l'ensemble, la performance est impressionnante. Pour l'avenir, cependant, la situation apparaît plus difficile. On a l'impression qu'à peu près toutes les avenues ont déjà été explorées et on voit mal comment les recettes du gouvernement pourraient être accrues au rythme où elles l'ont été au cours des dernières années, quoique la nonindexation de l'impôt sur le revenu laisse encore une certaine marge de manœuvre. Ceci nous amène à traiter des compressions budgétaires.

\section{Les compressions budgétaires}

On parle beaucoup de compression des dépenses. Avant toute autre chose, il importe de préciser par rapport à quoi l'on «comprime». Il convient donc d'examiner la ventilation des dépenses du gouvernement québécois et de la comparer à celle des autres gouvernements provinciaux. Les données sont présentées au tableau 3. Les dépenses affectées au service de la dette et aux transferts généraux aux administrations locales, qui ne reflètent pas les priorités sectorielles des gouvernements, sont exclues. Dans le reste du Canada, environ les deux tiers des dépenses sont consacrées à la santé, aux services sociaux et à l'éducation. L'Ontario se distingue par ses dépenses élevées dans le domaine de la santé. Simeon et Miller ${ }^{36}$ montrent que cette situation prévalait déjà en 1972: en général les provinces les plus urbanisées et les plus riches dépensent davantage dans ce domaine. À l'opposé, les fonds alloués aux ressources naturelles et à l'industrie sont limités, ce qui aussi n'est pas nou-

32. Richard Simeon et E. Robert Miller, «Regional Variations in Public Policy ", dans David J. Elkins et Richard Simeon (dit), Small Worlds: Provinces and Parties in Canadian Political Life. (Toronto, Methuen 1980). 
TABLEAU 3

La ventilation des dépenses des gouvernements provinciaux au Canada, 1979-80 ${ }^{1}$

\begin{tabular}{|c|c|c|c|c|}
\hline Fonction & $\begin{array}{c}\text { Québec } \\
\%\end{array}$ & $\begin{array}{c}\text { Reste } \\
\text { du Canada } \\
\%\end{array}$ & $\begin{array}{c}\text { Ontario } \\
\%\end{array}$ & $\begin{array}{c}\text { Sas- } \\
\text { katchewan } \\
\%\end{array}$ \\
\hline $\begin{array}{l}\text { Protection } \\
\text { Transport } \\
\text { Santé } \\
\text { Serv. sociaux } \\
\text { Éducation } \\
\text { Res. et indust. } \\
\text { Amén. régional } \\
\text { Autres }^{2}\end{array}$ & $\begin{array}{r}3.5 \\
7.2 \\
25.3 \\
16.2 \\
27.6 \\
4.7 \\
1.1 \\
14.5\end{array}$ & $\begin{array}{r}3.9 \\
8.8 \\
27.5 \\
14.9 \\
23.3 \\
7.2 \\
1.4 \\
13.0\end{array}$ & $\begin{array}{r}3.9 \\
7.9 \\
30.3 \\
16.1 \\
25.6 \\
4.1 \\
1.2 \\
10.9\end{array}$ & $\begin{array}{r}3.2 \\
8.4 \\
23.9 \\
15.8 \\
20.5 \\
15.3 \\
2.1 \\
10.7\end{array}$ \\
\hline & $100 \%$ & $100 \%$ & $100 \%$ & $100 \%$ \\
\hline
\end{tabular}

1. Les dépenses affectées au service de la dette et aux transferts à des fins générales aux administrations locales sont exclues de même que celles découlant du régime de rentes du Québec et du Canada.

2. Comprend les services généraux.

Sources: Statistique Canada, Les finances publiques provinciales, cat. 68-207.

veau, puisque ce sont «the less developed provinces which commit the most to promoting trade and industry ${ }^{33}$. Pour ce qui est de la Saskatchewan, l'aide à l'agriculture et à l'industrie pétrolière y est particulièrement élevée. Le fait que les fonds consacrés à la santé soient assez faibles renvoie probablement au caractère agricole de la province ${ }^{34}$ et non à un moins grand intérêt pour ce secteur. Au contraire la Saskatchewan a été la première province à adopter les programmes d'assurancehospitalisation et d'assurance-maladie.

Ces tendances jettent un éclairage différent sur la ventilation observée au Québec. Le gouvernement québécois dépense

33. Ibid., 271.

34. Joseph Eyes et Peter Sterling, «Stress Related Mortality and Social Organization». Review of Radical Political Economics 91, (printemps 1977): 1-45. 
davantage dans le domaine de l'éducation, ce qui représente un changement substantiel par rapport à 1972, année où le Québec arrivait en huitième place pour ce qui est de la proportion du budget consacrée à l'éducation ${ }^{35}$. C'est le secteur où le gouvernement québécois a investi le plus (en termes relatifs) dans la décennie 70. Par contre, le gouvernement du Québec consacre une plus faible proportion de son budget à la santé, au transport et au développement de l'industrie primaire et secondaire. Nous ne disposons pas d'information suffisante sur les raisons de cette situation ${ }^{36}$. Tout indique cependant que dans le cas de la santé et du transport c'est le résultat de décisions prises au milieu ou à la fin des années 70 . Dans le domaine de la santé, le Québec était au-dessus de la moyenne canadienne en 1972. Pour ce qui est du transport ce n'est que depuis l'arrivée au pouvoir du Parti Québécois que le Québec se retrouve derrière l'Ontario. Dans le secteur industriel (y compris les ressources), la tendance existait déjà au début des années 70 .

Les tendances d'arrière-plan étant dégagées, on peut revenir à la question des compressions budgétaires. Il faut d'abord préciser que le thème n'est pas nouveau. Dès son premier budget, le gouvernement péquiste parle de l'assainissement des finances publiques et de contrôle des dépenses ${ }^{37}$. Dans le second, le ministre des Finances fait état d'une «série de démarches exprimant fort bien le désir du gouvernement de forcer les ministères à «dégraisser»... un peu leur substance ${ }^{38}$. Le budget 1979-80 établit comme premier objectif le contrôle des dépenses de l'État ${ }^{39}$. Le budget 1980-81 consacre six pages à

35. Simeon et Miller, op. cit.

36. André Blais et Kenneth McRoberts, «Public Expenditure in Ontario and Quebec, 1950-1980: Explaining the Differences», communication présentée au colloque sur le développement de l'État au Québec et en Ontario, Montréal, (déc. 1981).

37. Gouvernement du Québec, Budget 1977-1978, Discours sur le budget, 23.

38. Gouvernement du Québec, Budget 1978-1979, Discours sur le budget, 23.

39. Gouvernement du Québec, Budget 1979-1980, Discours sur le budget, 21. 
ce même thème ${ }^{40}$. Dans le budget $1981-82$ on parle de la nécessité de «couper sérieusement le rythme d'augmentation des dépenses ${ }^{41}$. En 1982, le ministre des Finances annonce la poursuite des coupures ${ }^{42}$.

Le discours est très cohérent. Les chiffres sont cependant beaucoup moins clairs. Jusqu'en 1980, la part des dépenses dans le produit intérieur brut est à la hausse (tableau 1). Cette tendance s'est poursuivie jusqu'en 1982. Le budget 1982-83 prévoit une augmentation des dépenses de $11 \%$, ce qui correspond à la hausse anticipée du produit intérieur brut. Il est donc un peu étrange de parler de compression. Au total, les dépenses ont continué d'augmenter, avec une possibilité de plafonnement au cours de la dernière année.

Sur ce plan, l'évolution du Québec est fort différente de celle de l'Ontario. Dès 1975, le ministre des Finances de l'Ontario déclarait :

"I am convinced that one of the root causes of the current inflation problem in Canada is excessive government spending and unnecessary growth in the size and complexity of the public sector. This has shifted an increasing share of our total resources out of private production uses in the economy, and has eroded the taxpayer's hard-earned income. With this budget, therefore, Ontario continues and extends its tough measures to curb the growth of government ${ }^{43}$.

Il s'en est suivi une diminution relative des dépenses (tableau 1) et une réduction absolue de $5.7 \%$ (entre 1975 et 1981) ${ }^{44}$ des employés. Au Québec, les préoccupations pour le contrôle des dépenses apparaissent, on l'a vu, dans le budget de 1977. Mais les gestes concrets ont été assez timides. Depuis 1979, il

25.

40. Gouvernement du Québec, Budget 1980-1981, Discours sur le budget, 20-

41. Gouvernement du Québec, Budget 1981-1982, Discours sur le budget, 19.

42. Gouvernement du Québec, Budget 1982-1983, Discours sur le budget, 16.

43. Ontario, 1975-76 Budget, 16.

44. Le budget de l'Ontario, 1981-82, document budgétaire C, 5 . 
y a bien un gel des effectifs du secteur public. Il n'en demeure pas moins que, jusqu'à tout récemment, la croissance des dépenses s'est poursuivie. Le véritable mouvement de compression ne s'est enclenché qu’à partir de 1981. La non-indexation de l'impôt sur le revenu laissait, il faut le dire, une plus grande manœuvre au gouvernement québécois. Mais les contraintes fiscales, dont nous avons déjà parlé, ne pourraient être reportées indéfiniment. Le fardeau fiscal des Québécois devenant de plus en plus lourd, il devenait impératif de passer aux actes. Tout ceci suggère que le gouvernement péquiste, malgré tout ce qu'on a pu en dire, a été très réticent à réduire le rôle de l'État.

Mais, alors, ces fameuses compressions budgétaires dont le gouvernement et les médias nous parlent constamment ne sont-elles qu'un mythe? Pas vraiment. Il convient toutefois de rappeler que les changements ne sont pas unidirectionnels, que si certains services ou programmes sont réduits, d'autres connaissent par contre une expansion et même certains nouveaux programmes sont créés. Mais pourquoi parler de façon quasi-exclusive des compressions budgétaires? L'explication nous est donnée par le ministre des Finances dans son budget 1981-82:

«En première étape de la préparation du budget, on projette sur l'année suivante le coût des opérations et des programmes existants, sans rien y changer, mais en les ajustant simplement en fonction des taux prévus de salaires, d'inflation et d'intérêt. Puis on tient compte, selon le cas, des projections de clientèles et du coût pour une année entière, des mesures prises au cours de l'année précédente.

Cette vaste opération de projection purement mécanique aurait produit, en 1981-1982, une augmentation de plus de 19 pour cent des dépenses par rapport à 1980-1981». ${ }^{45}$

45. Gouvernement du Québec, Budget 1981-1982, Discours sur le budget, 27. 
La logique est simple. Même si le gouvernement ne modifie pas ses programmes, les dépenses augmentent sensiblement, parce que les coûts d'opération augmentent ou parce que la clientèle s'accroît. Les choix (par rapport au statu quo) ne peuvent alors qu'être pour l'essentiel, dans le sens d'une réduction. Pour clarifier les choses, il faut d'ailleurs distinguer les postes budgétaires pour lesquels le gouvernement dispose d'une certaine marge de manœuvre et ceux pour lesquels cette marge est à peu près nulle. Dans cette catégorie, on retrouve deux éléments principaux.

Le premier élément est le service de la dette. Le coût de la dette résulte de déficits encourus depuis vingt ans de même que des taux d'intérêts établis par la Banque du Canada. En 1982-83, les crédits prévus à ce poste sont presque le double de ceux de 1980-81, alors que l'ensemble des dépenses n'a augmenté pendant la même période que de $30 \%$. Il y a ainsi moins de place pour les dépenses «fonctionnelles».

Le deuxième élément, ce sont les conventions collectives. Les augmentations de salaire étaient dictées par la convention signée en 1979. La sécurité d'emploi rendait à peu près impossible toute réduction des effectifs. En 1981-82, le taux d'augmentation des salaires a été estimé à $14.7 \%$ par le ministre des Finances ${ }^{46}$ alors que le produit intérieur brut n'a progressé que de $12.7 \%$. Ces dépenses représentent environ la moitié du budget du gouvernement québécois. Les conséquences sont évidentes. Prenons le budget 1981-82, qui prévoyait des dépenses totales de $\$ 20$ milliards. La rémunération et le service de la dette absorbent environ $\$ 12$ milliards. Pour en arriver à une hausse globale de l'ordre de $13 \%$ du budget, l'ensemble des autres dépenses ne pouvait être accru que d'environ $8 \%$, ce qui est bien inférieur à l'inflation.

46. Gouvernement du Québec, Budget 1981-1982, Renseignements supplémentaires: crédits, 5. 
La question de la rémunération du secteur public mérite d'être examinée de plus près. Le débat a surtout porté sur la comparaison des salaires dans le secteur privé et le secteur public. Dans le budget 1982-83, le ministre des Finances parle d'un écart de $13 \%{ }^{47}$. On sait que ces données, provenant du Bureau de recherche sur la rémunération, ont été critiquées. D'autres études, employant une méthodologie différente, en arrivent cependant à des conclusions convergeantes, soit un écart de 10 à $15 \%^{48}$. Mais ce qui nous intéresse ici est différent. Il s'agit de comprendre pourquoi les dépenses découlant de l'application de la convention collective augmentent à un tel rythme. Sur ce point, un certain nombre de faits doivent être rappelés. D'une part, la convention signée en 1979 n'a rapporté que peu de modifications à la situation prévalant jusqu'alors. Essentiellement l'augmentation des salaires correspond à la hausse du coût de la vie. Pour l'ensemble de la convention, les salaires n'ont donc pas augmenté indûment: le ministre des Finances a noté d'ailleurs que l'écart avec le secteur privé a diminué depuis $1979^{49}$. D'autre part, un certain nombre de facteurs se sont conjugués pour rendre la situation particulièrement difficile à partir de 1981. Premièrement, une prime d'enrichissement collectif (de $1.2 \%$ en 1981 et de $1.6 \%$ en 1982) entre en vigueur à partir de ce moment, alors qu'il n'y en avait pas pour les deux premières années. En 1979, le gouvernement avait décidé de se montrer plus généreux pour la fin de la convention. Il en subit alors les conséquences. Deuxièmement, l'indexation étant calculée une année en retard, l'effet de l'inflation sur les finances publiques se manifeste avec

47. Gouvernement du Québec, Budget 1982-1983, Discours sur le budget, 18.

48. Robert Lacroix, "Les conséquences des disparités de salaires entre les secteurs privé et public au Québec", Miméo Bibliothèque Nationale du Québec, D82-962. 18.

49. Gouvernement du Québec, Budget 1982-1983, Discours sur le budget, 17- 
des délais. Prenons la période de juillet 1981 à juillet 1982. L'indice des prix à la consommation a augmenté d'environ $11 \%$ pendant cette période: l'indexation a cependant été calculée à partir de l'augmentation de l'indice au cours de la période précédente, qui était de $13 \%$. À cela s'ajoute la prime d'enrichissement collectif, ce qui donne une augmentation globale de $14 \%$ en 1981. Finalement il faut tenir compte du fait que les employés du secteur public sont relativement jeunes et que plusieurs peuvent encore monter un échelon dans l'échelle des salaires, ce qui accroît la masse salariale requise d'environ $2 \%$. Ces différents éléments ont fait que les salaires constituent une catégorie de dépenses particulièrement lourde depuis 1981. Cette évolution était toutefois prévisible.

La tentation était donc grande de réouvrir la convention collective de façon à réduire quelque peu les dépenses au titre de la rémunération. Le gouvernement a demandé aux syndicats d'annuler la hausse d'environ 11\%, prévue pour juillet 1982. Les syndicats ont refusé. Devant ce refus, les choix du gouvernement étaient limités. Augmenter encore plus le déficit était difficilement envisageable. Une hausse d'impôt s'avérait politiquement dangereuse, quoiqu'une hausse «déguisée» (la nonindexation totale de l'impôt sur le revenu des particuliers) aurait pu être envisagée. Le gouvernement aurait également pu procéder à des coupures encore plus radicales, impliquant l'abolition de programmes ou d'organismes, coupures auxquelles il fait maintenant allusion ${ }^{50}$. Le fait que cette possibilité n'ait pas été retenue nous semble significatif. Cela renforce l'argument selon lequel le gouvernement péquiste demeure favorable à l'intervention de l'État. Mais surtout, on en retire l'impression que le gouvernement était mal préparé à faire face à cette situation, qu'il ne semble pas avoir prévue.

50. La Presse, 9 novembre 1982. 
Le gouvernement a donc préféré tenter de récupérer en catastrophe l'augmentation consentie en juillet 1982 dans les premiers mois de l'année 1983. Il est évidemment facile à l'observateur, à posteriori, de critiquer une telle décision. Il faut bien reconnaittre que les autres possibilités (hausses d'impôt, coupures radicales) comportaient elles aussi des coûts politiques considérables. Stratégiquement, le gouvernement demande des «sacrifices» à un groupe bien limité et jouissant de la sécurité d'emploi. C'est d'ailleurs un des groupes qui a appuyé le plus massivement le Parti Québécois en 1981 (et au référendum) ${ }^{51}$. On peut cependant penser qu'une stratégie moins brutale (le gel des salaires de 1983) et plus diversifiée (la non-indexation totale, l'abolition de quelques programmes) aurait été moins coûteuse.

Mais revenons-en aux choix budgétaires du gouvernement. Nous avons établi qu'en général les dépenses gouvernementales n'étaient pas réduites mais qu'une hausse «mécanique» des coûts, qui échappe au contrôle du gouvernement, l'obligeait à procéder à des compressions au niveau des autres postes. Mais quelle est l'orientation générale des derniers budgets sur ce plan? Cette orientation peut-être dégagée en examinant la répartition des crédits prévue dans les budgets. Un tel exercice a cependant des limites, qu'il convient de préciser dès le départ. Premièrement, rien n'assure que les dépenses réelles correspondent aux crédits prévus. Ces derniers manifestent une intention, qui peut être modifiée en cours de route. Ce problème peut être corrigé (en partie) pour l'année 198182 , puisque nous disposons aussi des dépenses probables, telles qu'estimées lors du dépôt des crédits 1982-83. Deuxièmement, il est difficile de comparer les crédits d'une année à ceux de

51. André Blais et Richard Nadeau, «L'appui au Parti Québécois: évolution de la clientèle de 1970 et 1981 » et «L'appui au OUI», dans Jean Crête (dir.). Le comportement électoral au Québec, (Chicoutimi, G. Morin, à venir). 
l'année antérieure, à cause des contraintes «incontrôlables ». Le budget d'un ministère dont l'activité exige moins de capital humain ou dont la clientèle est en déclin est plus facilement compressible. L'idéal serait donc de comparer les crédits octroyés à ceux que donnerait la «projection mécanique» des coûts. Malheureusement, nous n'avons que peu d'information à ce sujet. Par contre, une publication du Conseil du Trésor fait état des orientations budgétaires dans les différents ministères en 1981-82 et 1982-83: nous y puiserons plusieurs informations utiles. Troisièmement, pour l'année 1982-83, les crédits annoncés le 23 mars ont été modifiés par le budget présenté en mai, qui retranche essentiellement $\$ 640$ millions au titre de la rémunération. Le budget ne précise toutefois pas la nouvelle répartition des crédits, sauf en ce qui concerne les ministères. Malgré tout, nous avons tenté d'isoler les principaux postes budgétaires qui ont vu les fonds qui leur sont alloués diminuer ou augmenter fortement (en dollard constants), de même que les programmes ou services qui y sont associés ${ }^{52}$.

Considérons d'abord le budget 1981-82. Les programmes les plus touchés par les compressions sont les suivants: l'Office franco-québécois pour la jeunesse, l'aide aux agriculteurs (qui sera cependant majorée en 1982), l'ensemble du ministère des Consommateurs, Coopérative et Institutions financières, la formation des adultes, l'Office des professions du Québec, l'aide à l'isolation des maisons, l'assainissement des eaux usées (les subventions augmentent cependant substantiellement en 1982), l'ensemble du ministère de l'Immigration, les loisirs, le service routier, les programmes de création d'emploi. À l'opposé, certains programmes ont vu les fonds qui leur sont oc-

52. L'exercice demeure bien approximatif. Les données sont d'ailleurs plus complètes pour l'année 1982-1983, pour laquelle le Conseil du Trésor indique pour chaque ministère la nature des compressions. Voir Gouvernement du Québec, Budget 1982-1983, Renseignements supplémentaires: crédits. 
troyés augmenter substantiellement: Radio-Québec (pour l'élargissement du réseau), le programme d'aide aux équipements publics (terminé en 1981), l'aide à l'habitation, l'aide à l'industrie forestière (dans le cadre d'une entente fédéraleprovinciale signée en 1979), l'aide à l'industrie manufacturière, la sécurité publique (suite au renouvellement de la convention des agents de la paix et de celle de la Sûreté du Québec).

Trois tendances semblent ressortir. Premièrement les coupures les plus importantes touchent des programmes marginaux (la protection du consommateur, l'immigration, les loisirs) ${ }^{53}$ ou qui sont en déclin depuis plusieurs années (le transport). Les «gros» programmes sont épargnés. Les mesures d'aide sociale sont peu modifiées. Il en est de même des services de santé. Deuxièmement, à peu près aucun programme n'a été aboli. Il aurait été en effet possible de maintenir le niveau de service dans la plupart des programmes en en éliminant quelques-uns, de façon plus sélective. Le gouvernement prétendait ainsi couper dans le «gras». Ce type de discours, qui ne se prête à aucune vérification empirique (comment, en effet, distinguer véritablement le «gras» du «maigre»?), avait évidemment pour but de justifier les décisions prises. Mais il a aussi comme effet «secondaire» de répandre l'image d'une administration publique inefficace. Troisièmement, certaines catégories de dépenses sont à la hausse. Dans la plupart des cas, toutefois, elles sont le résultat de décisions prises antérieurement. Certains choix sont cependant plus discutables: l'élargissement du réseau de Radio-Québec, par exemple, est-il si prioritaire, en pleine «crise»? Finalement le secteur de l'aide à l'industrie échappe complètement à l'effort de compression à

53. La culture est cependant en bonne partie épargnée, ce qui est en soi significatif. Le Parti Québécois est en effet souvent associé aux "travailleurs du langage». Voir Marcel Fournier, «La question nationale: enjeux et impasse» dans J.F. Léonard (dir.), La chance au coureur, (Montréal, Éditions Nouvelle Optique, 1978). 
un moment où l'on se préoccupe particulièrement de la relance de l'économie.

Lors du dépôt des crédits 1982-83, des coupures de l'ordre de $\$ 700$ millions sont annoncées. Un seul secteur est exempté: «Pour la seconde année consécutive les programmes de transfert de la mission économique ne sont pas assujettis à la règle des compressions budgétaires ${ }^{54}$. Deux autres reçoivent des fonds additionnels: les programmes de création d'emplois et l'aide à l'habitation Ces choix reflètent la préoccupation du gouvernement pour l'emploi et l'investissement. Pour le reste, à peu près tous les programmes sont réduits quelque peu. Les compressions les plus sensibles touchent les loisirs et le transport. Mais l'aide sociale est aussi visée. Les allocations familiales de base ne sont pas indexées, la liste de médicaments disponibles aux assistés-sociaux est rétrécie et des mesures sont adoptées pour exercer un plus grand contrôle sur les revenus des bénéficiaires. Les services dentaires aux enfants sont réduits (les services préventifs étant confiés aux C.L.S.C.), les barèmes d'admissibilité à l'aide juridique ne sont pas indexés, les loyers dans les H.L.M. sont augmentés. Dans chaque cas, il s'agit cependant de mesures plutôt marginales qui ne remettent pas en cause l'essentiel de l'intervention gouvernementale. Les compressions demeurent peu sélectives. À peu près aucune activité n'est abolie. L'aide à l'industrie est accrue. Par contre, on commence à toucher un peu plus les programmes de redistribution qui sont au coeur même de l'État-providence, en essayant de contrôler certains coûts (on pense en particulier à la consommation des médicaments, aux loyers dans les H.L.M., qui n'avaient pas été majorés depuis plusieurs années), alors qu'en 1981 , on avait visé davantage des programmes plus marginaux.

54. Gouvernement du Québec, Budget 1982-1983, Renseignements supplémentaires: crédits, 6 . 
Sur l'ensemble des deux dernières années, ce sont les secteurs du loisir et du transport qui semblent avoir subi les plus fortes compressions. Dans le secteur du transport, en particulier, les coupures sont considérables, si l'on compare avec ce qui se produit dans les autres provinces canadiennes. Par contre, l'aide à l'industrie a été complètement épargnée. Il faut préciser que c'est là un domaine où l'effort du Québec est inférieur à la moyenne canadienne, et qui a connu un certain déclin, à la fin des années $70^{55}$. Finalement, les dépenses pour l'éducation, ont été peu touchées, surtout à cause des contraintes qu'imposait la convention collective. Ce sera là un secteur clé à surveiller, puisque c'est celui où le Québec s'est le plus démarqué des autres provinces dans les années 70 . Une étude du gouvernement démontrait que les dépenses par étudiant étaient plus élevées de $\$ 605$ au Québec par rapport à l'Ontario, en 1979-80, alors qu'elles étaient inférieures de $\$ 105$ en $1972-73^{56}$

Les compressions budgétaires sont venues tardivement au Québec surtout si l'on compare à ce qu'a connu l'Ontario. La résistance à ces compressions est aussi beaucoup plus forte, l'État y ayant selon toute vraisemblance une plus grande légitimité. Les contraintes fiscales aidant, l'ère des «coupures» est cependant arrivée. Tout indique que le gouvernement québécois était peu préparé à ce nouveau contexte, le climat préréférendaire disposant davantage aux largesses. La récupération des salaires dans le secteur public s'est faite en catastrophe. Pour le reste, les compressions sont généralisées mais plutôt timides et n'affectent guère l'orientation générale des dépenses.

55. Philippe Faucher, André Blais et Robert Young, L'aide financière directe au secteur manufacturier: Québec et Ontario (1960-1980). (note de recherche, département de science politique, Université de Montréal, à venir).

56. Marius Demers, Les efforts financiers en éducation: une comparaison QuébecOntario. 1972-1973 à 1979-1980. (Québec, Ministère de l'Éducation, janvier 1982). 


\section{Conclusion}

Jusqu'à maintenant la question de l'intervention gouvernementale était posée en termes simples: la gauche était favorable à la croissance de l'État et la droite y était opposée. Il en est résulté que les dépenses de l'État (relatives au produit intérieur brut) augmentaient en moyenne de près de un pour cent quand le gouvernement était de gauche. Cette croissance de l'État a favorisé une certaine redistribution des revenus, sans restreindre vraiment la croissance économique. Certains indices suggèrent toutefois que, dans certains pays, les limites ont été atteintes. Dans les années 70, par exemple, une augmentation de l'impôt personnel (relatif au produit intérieur brut) de un pour cent semble avoir entrainé une diminution de $0.4 \%$ de l'investissement ${ }^{57}$. Cette nouvelle réalité fait en sorte que même la gauche est amenée à s'interroger sur la pertinence de l'intervention gouvernementale. Rosanvallon, par exemple, affirme que le projet socialiste doit s'inscrire «dans un triple mouvement de réduction de la demande d'État, de réencastrement de la solidarité dans la société et de production d'une plus grande visibilité sociale ${ }^{58}$. La croissance de l'État n'est plus considérée comme un objectif en soi.

L'État est ainsi soumis à de fortes pressions contradictoires. D'une part, les coûts des services qu'il offre ont tendance à augmenter. Dans le domaine de la santé, pour ne donner qu'un exemple, le vieillissement de la population ne peut qu'entrấner une augmentation de la demande de services. D'autre part, en période de croissance nulle, la résistance des contribuables se fait plus grande. Ce sera peut-être l'occasion de réfléchir de

57. Voir David Cameron, "The Expansion of the Public Economy». American Political Science Review 72, (déc. 1978): 1243-1262 et "On the Limits of the Public Economy». Annals of the American Academy of Political and Social Science, vol. 459, (janvier 1982): 46-63.

58. Pierre Rosanvallon, La crise de l'État-providence, (Paris, Seuil, 1981). 
façon plus large sur les objectifs et les limites de l'intervention gouvernementale. Pourquoi, d'ailleurs, ne penser qu'en termes de cpmpressions budgétaires? En voulant «couper» un peu partout sans «faire trop mal», on étouffe tout effort d'imagination. Le moment est peut-être venu de remettre en question certains programmes qui, tout en étant utiles, sont coûteux et exigent une lourde bureaucratie, de façon à dégager des fonds pour restructurer et améliorer des services jugés prioritaires. 